\title{
Complex Langevin study for polarons in a one-dimensional two-component Fermi gas with attractive contact interactions
}

\author{
Takahiro M. Doi, ${ }^{1, *}$ Hiroyuki Tajima $\odot,{ }^{2, \dagger}$ and Shoichiro Tsutsui ${ }^{3, \dagger}$ \\ ${ }^{1}$ Research Center for Nuclear Physics (RCNP), Osaka University, Osaka 567-0047, Japan \\ ${ }^{2}$ Department of Physics, Graduate School of Science, The University of Tokyo, Tokyo 113-0033, Japan \\ ${ }^{3}$ Theoretical Research Division, Nishina Center, RIKEN, Wako, Saitama 351-0198, Japan
}

(Received 27 May 2021; revised 24 June 2021; accepted 28 June 2021; published 20 August 2021)

\begin{abstract}
We investigate a polaronic excitation in a one-dimensional spin-1/2 Fermi gas with contact attractive interactions, using the complex Langevin method, which is a promising approach to evade a possible sign problem in quantum Monte Carlo simulations. We found that the complex Langevin method works correctly in a wide range of temperature, interaction strength, and population imbalance. The Fermi polaron energy extracted from the two-point imaginary Green's function is not sensitive to the temperature and the impurity concentration in the parameter region we considered. Our results show a good agreement with the solution of the thermodynamic Bethe ansatz at zero temperature.
\end{abstract}

DOI: 10.1103/PhysRevResearch.3.033180

\section{INTRODUCTION}

The quantum Monte Carlo method [1,2] is widely used in various fields of physics as a nonperturbative tool of analysis. In a path-integral formalism using the Lagrangian, a partition function is written in terms of the integral of the Boltzmann weight $e^{-S}$ over field variables, where $S$ is an action. When the action is a real-valued function, the Boltzmann weight is regarded as a probability density function. This ensures that the quantum expectation values of physical observables can be estimated by importance sampling of the Boltzmann weight. However, the positivity of the Boltzmann weight is violated in many physically interesting systems: the Hubbard model, finite density quantum chromodynamics (QCD), QCD with a $\theta$ term, matrix superstring models, and any systems defined by the Schwinger-Keldysh formalism which describes real-time dynamics, for instance [3-10]. In these cases, the number of samples becomes exponentially large as the system size grows in order to obtain statistically significant results. In nonrelativistic fermionic systems, a frequently used way to apply the quantum Monte Carlo method is by introducing a bosonic auxiliary field through the Hubbard-Stratonovich transformation [11-14]. After integrating out the fermion fields, we will obtain an effective action of the auxiliary field. Since the effective action involves a logarithm of a fermion determinant, the positivity is not guaranteed except in a few cases where the

\footnotetext{
*takahiro.doi@rcnp.osaka-u.ac.jp

†hiroyuki.tajima@phys.s.u-tokyo.ac.jp

"shoichiro.tsutsui@ riken.jp
}

Published by the American Physical Society under the terms of the Creative Commons Attribution 4.0 International license. Further distribution of this work must maintain attribution to the author(s) and the published article's title, journal citation, and DOI. action has particle-hole symmetry [15], Kramers symmetry [16], or Majorana positivity [17-19], for instance.

A promising approach to evade the sign problem is the complex Langevin method [20,21], which is an extension of the stochastic quantization to systems with complex-valued actions. An advantage of this method is that it is scalable to the system size, and thus the computational cost is similar to the usual quantum Monte Carlo method without the sign problem. On the other hand, it is known that this method sometimes gives incorrect answers even when the statistical average of a physical observable converges. In the past decade, a way to judge the reliability of the complex Langevin method has been extensively studied [22-32], and criteria were proposed which are able to compute in actual simulations using the boundary terms $[22,23,30,31]$ and the probability distribution of the drift term $[24,26]$. While it is still difficult to predict when the complex Langevin method fails without performing numerical simulations, we can eliminate wrongly convergent results owing to these criteria. In the context of cold fermionic atoms, the complex Langevin method is applied to rotating bosons [33], polarized fermions [34-37], unpolarized fermions with contact repulsive interactions [38], and mass imbalanced fermions [39] to study the ground-state energy, thermodynamic quantities, and Fulde-Ferrell-Larkin-Ovchinnikov-type pairings (see also a recent review [40]).

In this paper, we consider spatially one-dimensional spin$1 / 2$ polarized fermions with contact attractive interactions which is known as the Gaudin-Yang model [41], and compute the single-particle energy of spin-down fermions in a spin-up Fermi sea, which is referred to as the Fermi polaron energy. Recently, the single-particle excitation spectra of Fermi polarons were experimentally measured in higher-dimensional atomic systems [42-49] (also see a recent review [50] for Fermi polarons). While an analytic formula for the polaron energy in one dimension is obtained exactly at zero temperature based on the thermodynamic Bethe ansatz method [51], 
no analytical solutions are known at finite temperatures (note that a Fredholm determinant representation of the momentum distribution and the two-point Green's function of the polaron is studied in Refs. [52-54], and numerical results of the thermodynamic properties within the thermodynamic Bethe ansatz are reported in Ref. [55]). One-dimensional Fermi polarons were studied with several theoretical approaches such as the Bruckner-Hartree-Fock [56], $T$-matrix [57,58], and variational $[59,60]$ approaches. Note that Fermi polarons in higher dimensions have also been investigated by a diagrammatic series expansion called the diagrammatic Monte Carlo method [61,62]. In this study, we demonstrate that a microscopic quantity, that is, the polaron energy, is efficiently computed by the complex Langevin method.

This paper is organized as follows. In Sec. II, we derive a lattice action of the Gaudin-Yang model. In Sec. III, we review how to compute physical quantities using the complex Langevin method. In Sec. IV, we show a way to extract the ground-state energy in the spin-down channel from a twopoint imaginary time Green's function. In Sec. V, we present the numerical results. Section VI is devoted to the summary of this paper. In this work, $k_{\mathrm{B}}$ and $\hbar$ are taken to be unity.

\section{THE GAUDIN-YANG MODEL}

We consider a one-dimensional two-component Fermi gas with contact attractive interactions which is known as the
Gaudin-Yang model $[63,64]$. The Hamiltonian is given by

$$
\begin{aligned}
\hat{H}= & \sum_{p, \sigma}\left(\frac{p^{2}}{2}-\mu_{\sigma}\right) \hat{c}_{p, \sigma}^{\dagger} \hat{c}_{p, \sigma} \\
& -g \sum_{p, p^{\prime}, q} \hat{c}_{p+\frac{q}{2}, \uparrow}^{\dagger} \hat{c}_{-p+\frac{q}{2}, \downarrow}^{\dagger} \hat{c}_{-p^{\prime}+\frac{q}{2}, \downarrow} \hat{c}_{p^{\prime}+\frac{q}{2}, \uparrow},
\end{aligned}
$$

where $\hat{c}_{p, \sigma}$ and $\hat{c}_{p, \sigma}^{\dagger}$ are fermionic annihilation/creation operators with momentum $p$ and spin $\sigma=\uparrow, \downarrow$, respectively. In this work, the atomic mass is taken to be unity. The coupling constant $g$ is related to a scattering length in one dimension $a$ as $g=\frac{2}{a}>0$ [41]. The chemical potentials of spin- $\sigma$ fermions are represented by $\mu_{\sigma}$. For convenience, we introduce an average chemical potential $\mu=\left(\mu_{\uparrow}+\mu_{\downarrow}\right) / 2$ and a fictitious Zeeman field $h=\left(\mu_{\uparrow}-\mu_{\downarrow}\right) / 2$. The grand canonical partition function is given by $Z=\operatorname{Tr}\left[e^{-\beta\left(\hat{H}-\sum_{\sigma} \mu_{\sigma} \hat{N}_{\sigma}\right)}\right]$ with $\beta$ being an inverse temperature and a number operator $\hat{N}_{\sigma}=\sum_{p} \hat{c}_{p, \sigma}^{\dagger} \hat{c}_{p, \sigma}$. The path-integral representation of $Z$ reads

$$
Z=\int \prod_{\sigma} \mathcal{D} \psi_{\sigma}^{*} \mathcal{D} \psi_{\sigma} e^{-S}
$$

where action $S$ is given by

$$
S=\int_{0}^{\beta} d \tau \int d x\left[\sum_{\sigma=\uparrow, \downarrow} \psi_{\sigma}^{*}(x, \tau)\left(\frac{\partial}{\partial \tau}-\frac{1}{2} \frac{\partial^{2}}{\partial x^{2}}-\mu_{\sigma}\right) \psi_{\sigma}(x, \tau)-g \psi_{\uparrow}^{*}(x, \tau) \psi_{\downarrow}^{*}(x, \tau) \psi_{\downarrow}(x, \tau) \psi_{\uparrow}(x, \tau)\right] .
$$

Here, $\psi_{\sigma}(x, \tau), \psi_{\sigma}^{*}(x, \tau)$ are a Grassmann field and its complex conjugate.

While the action (3) is given in a continuous spacetime, one should perform a lattice regularization appropriately to carry out numerical simulations. We write the lattice spacing of the temporal and spatial directions as $a_{\tau}$ and $a_{x}$, respectively, and their ratio as $r=a_{\tau} / a_{x}^{2}$. We also introduce lattice quantities as

$$
\bar{\mu}_{\sigma} \equiv \mu_{\sigma} a_{x}^{2}, \quad \bar{g} \equiv g a_{x}, \quad \bar{\psi}_{\sigma, j, n} \equiv \psi_{\sigma}\left(j a_{x}, n a_{\tau}\right) a_{x}^{1 / 2},
$$

where $n$ and $j$ are integers that satisfy $0 \leqslant n<N_{\tau}$ and $0 \leqslant j<N_{x}$. The inverse temperature and the spatial length of the lattice is given by $\beta=T^{-1}=N_{\tau} a_{\tau}$ and $L=N_{x} a_{x}$. With these notations, we consider a lattice action,

$$
S_{\text {lat }}=\sum_{j, n} \sum_{\sigma=\uparrow, \downarrow}\left(\bar{\psi}_{\sigma ; j, n}^{*} \bar{\psi}_{\sigma ; j, n}-\bar{\psi}_{\sigma ; j, n+1}^{*} e^{-\bar{\phi}_{j, n}+\bar{\mu}_{\sigma}} \bar{\psi}_{\sigma ; j, n}+\frac{r}{2}\left(\bar{\psi}_{\sigma ; j+1, n}^{*}-\bar{\psi}_{\sigma ; j, n}^{*}\right)\left(\bar{\psi}_{\sigma ; j+1, n}-\bar{\psi}_{\sigma ; j, n}\right)\right)+\sum_{j, n} \frac{\cosh \left(\bar{\phi}_{j, n}\right)-1}{\bar{g}},
$$

where $\bar{\phi}_{j, n}$ is a bosonic auxiliary field. As shown in Ref. [14], the lattice action (5) correctly converges to the continuum one as long as the matching conditions

$$
\frac{g a_{\tau}}{a_{x}}=\left(\frac{f_{2}}{f_{0}}-\frac{f_{1}^{2}}{f_{0}^{2}}\right) e^{\bar{\mu}_{\uparrow}+\bar{\mu}_{\downarrow}}, \quad \mu_{\sigma} a_{\tau}=\frac{f_{1}}{f_{0}} e^{\bar{\mu}_{\sigma}}-1
$$

are satisfied, where $f_{k}$ is a $\bar{g}$-dependent constant given by

$$
f_{k}=\int_{-\infty}^{\infty} d \bar{\phi} e^{-\frac{\cosh (\bar{\phi})-1}{\bar{s}}} e^{k \bar{\phi}}
$$

In practice, it is sufficient to use an approximated form of the matching conditions

$$
\bar{g} \simeq \frac{g a_{\tau}}{a_{x}}, \quad \bar{\mu}_{\sigma} \simeq \mu_{\sigma} a_{\tau}-\frac{g a_{\tau}}{2 a_{x}}
$$

which are obtained as the first-order approximation in the expansion in terms of $a_{\tau}$. After integrating out the fermion fields, the partition function and the effective action of the auxiliary field read

$$
Z=\int \prod_{j, n} d \bar{\phi}_{j, n} e^{-S_{\mathrm{eff}}[\bar{\phi}]}
$$


where the effective action of the auxiliary field is given by

$$
\begin{aligned}
S_{\mathrm{eff}}[\bar{\phi}]= & \sum_{j, n} \frac{\cosh \bar{\phi}_{j, n}-1}{\bar{g}} \\
& -\sum_{\sigma} \log \operatorname{det}\left[I+e^{N_{\tau} \bar{\mu}_{\sigma}} B^{-1} C_{N_{\tau}-1} \cdots B^{-1} C_{0}\right] \\
B_{j, j^{\prime}} & =-\frac{r}{2}\left(\delta_{j-1, j^{\prime}}+\delta_{j+1, j^{\prime}}\right)+(1+r) \delta_{j, j^{\prime}} \\
\left(C_{n}\right)_{j, j^{\prime}} & =\delta_{j, j^{\prime}} e^{-\bar{\phi}_{j, n}}
\end{aligned}
$$

where $I$ is the $N_{x} \times N_{x}$ identity matrix. Since we consider a naive finite difference as an approximation of the secondorder derivative with respect to $x$, the eigenvalues of $B$ are $1+2 r \sin ^{2} \frac{\pi k}{N_{x}}\left(k=0,1, \ldots, N_{x}-1\right)$. It has been argued in Refs. $[11,14]$ that this naive lattice action converges too slowly to the continuum limit, and the behavior can be improved by replacing the eigenvalues of $B$ with

$$
\lambda_{k}=\exp \left[\frac{r}{2}\left(\frac{2 \pi k}{N_{x}}\right)^{2}\right]
$$

After this replacement, the form of $B$ is given by

$$
B_{j, j^{\prime}}=\frac{1}{N_{x}} \sum_{k=-\left\lfloor N_{x} / 2\right\rfloor}^{\left\lfloor N_{x} / 2\right\rfloor} \lambda_{k} \cos k\left(j-j^{\prime}\right) .
$$

A notable point is that the effective action (10) involves a logarithm of the fermion determinant which can be complex in general. Therefore, this term may cause the sign problem if the Zeeman field $h$ is not zero and then the Monte Carlo simulation can be difficult to apply to this system.

\section{COMPLEX LANGEVIN METHOD}

The complex Langevin method (CLM) [20,21] is an extension of the stochastic quantization which is usually applicable to real-valued actions. In the CLM, we first consider a complexified auxiliary field $\bar{\phi}_{n, k}$ and extend the domain of definition of $S_{\text {eff }}$ to the complex space. For such a complex field, we consider a fictitious time evolution described by the complex Langevin equation

$$
\bar{\phi}_{n, k}^{\eta}(t+\Delta t)=\bar{\phi}_{n, k}^{\eta}(t)-\frac{\partial S_{\text {eff }}}{\partial \bar{\phi}_{n, k}^{\eta}} \Delta t+\eta_{n, k}(t) \sqrt{\Delta t},
$$

where $\eta_{n, k}(t)$ is a real Gaussian noise. When we assume that the system described by the complex Langevin equation reaches equilibrium at $t=t_{\mathrm{eq}}$, an average of a physical observable $O(\bar{\phi})$ can be defined as

$$
\langle O(\bar{\phi})\rangle \equiv \lim _{T \rightarrow \infty} \frac{1}{T} \int_{t_{\mathrm{eq}}}^{t_{\mathrm{eq}}+T} d t\left\langle O\left(\bar{\phi}^{\eta}(t)\right)\right\rangle_{\eta},
$$

with the average over the noise $\left\langle O\left(\bar{\phi}^{\eta}(t)\right)\right\rangle_{\eta}$ being

$$
\left\langle O\left(\bar{\phi}^{\eta}(t)\right)\right\rangle_{\eta} \equiv \frac{\int \prod_{n, k, t} d \eta_{n, k}(t) O\left(\bar{\phi}^{\eta}(t)\right) e^{-\frac{1}{4} \sum_{n, k, t} \eta_{n, k}(t)^{2}}}{\int \prod_{n, k, t} d \eta_{n, k}(t) e^{-\frac{1}{4} \sum_{n, k, t} \eta_{n, k}(t)^{2}}} .
$$

We note that $\left\langle\eta_{n, k}(t) \eta_{n^{\prime}, k^{\prime}}\left(t^{\prime}\right)\right\rangle_{\eta}=2 \delta_{n n^{\prime}} \delta_{k k^{\prime}} \delta_{t t^{\prime}}$, in particular. Although we expect that the mean value $\langle O(\bar{\phi})\rangle$ is equivalent to the quantum expectation value calculated in an original action, i.e., $\int \prod_{n, k} d \bar{\phi}_{n, k} O(\bar{\phi}) e^{-S_{\text {eff }}} / \int \prod_{n, k} d \bar{\phi}_{n, k} e^{-S_{\text {eff }}}$ in the limit $\Delta t \rightarrow 0$, it is not correct in general. There are extensive studies [22-32] to understand when the CLM is justified, and criteria for determining whether a CLM is reliable or not have been proposed. One of a practical criterion which can be relied on in actual numerical simulations is discussed from the viewpoint of a probability distribution of a drift term [24,26]. In our case, it is sufficient to consider a magnitude of the drift term given by

$$
v^{\eta} \equiv \max _{n, k}\left|\frac{\partial S_{\text {eff }}}{\partial \bar{\phi}_{n, k}^{\eta}}\right|
$$

and its distribution. According to the criterion, the CLM is reliable if the probability distribution of $v^{\eta}$ shows an exponential decay.

\section{OBSERVABLES}

The number density of spin- $\sigma$ fermions is given by

$$
\begin{aligned}
n_{\sigma}= & \frac{T}{L} \frac{\partial}{\partial \mu_{\sigma}} \log Z=\frac{1}{L} \frac{1}{Z} \int \prod_{j, n} d \bar{\phi}_{j, n} \\
& \times \operatorname{tr}\left[\frac{1}{I+e^{-N_{\tau} \bar{\mu}_{\sigma}} C_{0}^{-1} B \cdots C_{N_{\tau}-1}^{-1} B}\right] e^{-S_{\text {eff }[\bar{\phi}]} .}
\end{aligned}
$$

The particle number density on a lattice unit is defined by $\bar{n}_{\sigma}=n_{\sigma} a_{x}$. From below, we assume that the spin-down fermions are regarded as a minority. Typical temperature and momentum scales are given by the Fermi scales which are determined by the density of spin-up fermions:

$$
T_{\mathrm{F}}=\frac{\pi^{2} n_{\uparrow}}{2}, \quad p_{\mathrm{F}}=\pi n_{\uparrow} .
$$

In lattice simulations, we can compute dimensionless combinations $T / T_{\mathrm{F}}$ and $p_{\mathrm{F}} a$ as follows:

$$
\frac{T}{T_{\mathrm{F}}}=\frac{2}{\pi^{2} \bar{n}_{\uparrow}^{2} N_{\tau} r}, \quad p_{\mathrm{F}} a=\frac{2 \pi r \bar{n}_{\uparrow}}{\bar{g}} .
$$

In order to calculate the polaron energy, we consider the two-point Green's function,

$$
G(p, \tau) \equiv \frac{1}{Z} \operatorname{Tr}\left[e^{-\beta \hat{K}} \mathrm{~T}_{\tau}\left[\hat{c}_{\downarrow, p}^{\dagger}(\tau) \hat{c}_{\downarrow, 0}(0)\right]\right] \quad(-\beta \leqslant \tau \leqslant \beta),
$$

where $\mathrm{T}_{\tau}$ is the imaginary-time-ordered product. Hereinafter we restrict $\tau>0$. We write the eigenvalue and eigenstate of $\hat{K}$ by $\hat{K}|n\rangle=K_{n}|n\rangle$. In particular, $K_{0}<K_{1}<\cdots$. We also assume that the ground state $|0\rangle$ is not degenerate. Expanding the trace by the eigenstates, the correlation function reads

$$
G(p, \tau)=\frac{\sum_{n m} e^{-(\beta-\tau) \Delta K_{n}-\tau \Delta K_{m}}\left\langle n\left|\hat{c}_{\sigma, p}^{\dagger}\right| m\right\rangle\left\langle m\left|\hat{c}_{\sigma^{\prime}, p^{\prime}}\right| n\right\rangle}{\sum_{n} e^{-\beta \Delta K_{n}}},
$$

where $\Delta K_{n} \equiv K_{n}-K_{0}$. In the low-temperature limit $\beta \rightarrow \infty$, only the ground state contributes to the summation over $n$. 


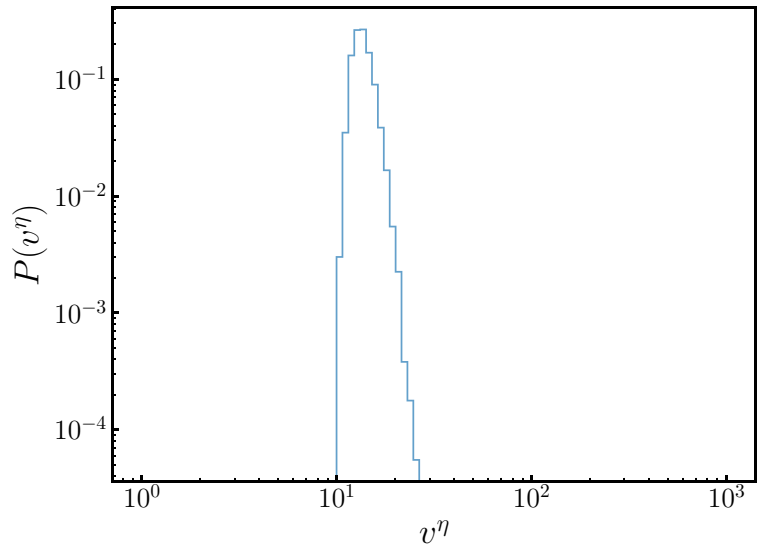

FIG. 1. The histogram of the drift term for the $N_{\tau}=40$ lattice at $\beta \mu=0, \beta h=12$.

Thus, we find

$\tilde{G}(p, \tau) \equiv \lim _{\beta \rightarrow \infty} G(p, \tau)=\sum_{m} e^{-\tau \Delta K_{m}}\left\langle 0\left|\hat{c}_{\sigma, p}^{\dagger}\right| m\right\rangle\left\langle m\left|\hat{c}_{\sigma^{\prime}, p^{\prime}}\right| 0\right\rangle$.

Since the matrix elements appearing in the above expression do not depend on $\tau$, the correlation function behaves as

$$
\tilde{G}(p, \tau)=A_{0} e^{-\tau E_{0}}+A_{1} e^{-\tau E_{1}}+\cdots,
$$

where $A_{0}, A_{1}, \ldots$ are $\tau$-independent constants, and $E_{0}, E_{1}, \ldots$ are energies of the ground state and excited states. In particular, the energy of the ground state can be

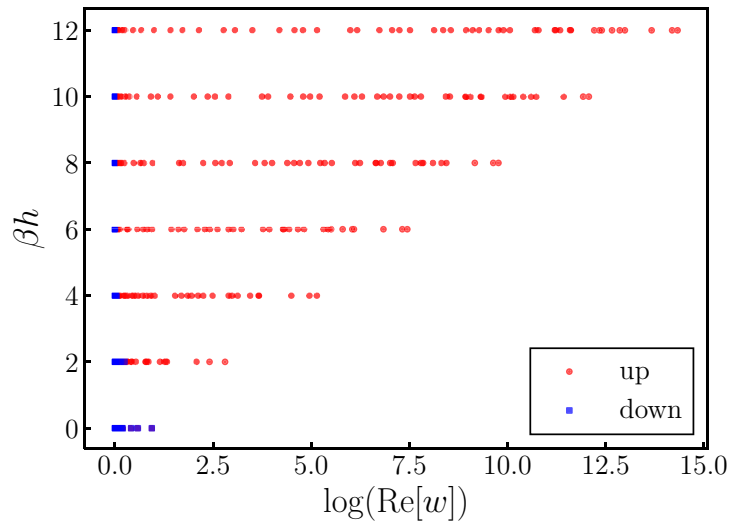

FIG. 2. The eigenvalues of matrix $G_{\sigma, \text { red }}^{-1}$ with several values of $h$ in the case of $N_{\tau}=40$ and $\beta \mu=-1.2$. Red circles and blue squares correspond to $G_{\uparrow, \text { red }}^{-1}$ and $G_{\downarrow, \text { red }}^{-1}$, respectively.

extracted by

$$
E_{0}(p)=\frac{1}{a_{\tau}} \lim _{\tau \rightarrow \infty} R(p, \tau), \quad R(p, \tau) \equiv \log \frac{\tilde{G}(p, \tau)}{\tilde{G}\left(p, \tau+a_{\tau}\right)},
$$

keeping $\tau \ll \beta$. The polaron energy $U$ is defined by

$$
U \equiv E_{0}(0)+\mu_{\downarrow} .
$$

The polaron energy is the shift of single-particle energy from that in the case of free fermions due to the interaction between the majority (spin-up fermions) and minority (spin-down fermions). We note that the polaron energy at zero temperature is calculated exactly based on the thermodynamic Bethe ansatz [51]:

$$
\frac{U}{T_{\mathrm{F}}}=-\frac{2}{\pi}\left\{\frac{1}{p_{\mathrm{F}} a}+\tan ^{-1}\left(\frac{1}{p_{\mathrm{F}} a}\right)+\left[\frac{\pi}{2}+\tan ^{-1}\left(\frac{1}{p_{\mathrm{F}} a}\right)\right] \frac{1}{\left(p_{\mathrm{F}} a\right)^{2}}\right\} .
$$

In lattice calculations, the polaron energy is obtained as follows. From the form of the effective action, the lattice expression of the inverse Green's function reads

$$
G^{-1} \equiv\left(\begin{array}{cccccc}
B & 0 & 0 & \cdots & 0 & e^{\bar{\mu}_{\downarrow}} C_{N_{\tau}-1} \\
-e^{\bar{\mu}_{\downarrow}} C_{0} & B & 0 & \cdots & 0 & 0 \\
0 & -e^{\bar{\mu}_{\downarrow}} C_{1} & B & \cdots & 0 & 0 \\
\vdots & \vdots & \vdots & \ddots & \vdots & \vdots \\
0 & 0 & 0 & \cdots & -e^{\bar{\mu}_{\downarrow}} C_{N_{\tau}-2} & B
\end{array}\right) .
$$

From straightforward algebra, each component of $G$ reads

$$
\begin{gathered}
2 G_{j j}=\frac{B^{-1}}{I+e^{N_{\tau} \bar{\iota}_{\downarrow}} B^{-1} C_{j-1} \cdots B^{-1} C_{0} B^{-1} C_{N_{\tau}-1} \cdots B^{-1} C_{j}}, \\
G_{k j}= \begin{cases}B^{-1} C_{k-1} \cdots B^{-1} C_{j} G_{j j} & (j+1 \leqslant k \leqslant N-1), \\
-B^{-1} C_{k-1} \cdots B^{-1} C_{0} B^{-1} C_{N-1} \cdots B^{-1} C_{j} G_{j j} & (0 \leqslant k \leqslant j-1) .\end{cases}
\end{gathered}
$$

The momentum representation of $G_{i j}$ is calculated by the discrete Fourier transformation. Therefore, if the temporal lattice size $N_{\tau}$ is sufficiently large, $\tilde{G}(p, \tau)$ is approximately given by

$$
\tilde{G}\left(2 \pi k / N_{x}, n a_{\tau}\right) \simeq \frac{1}{N_{x}} \sum_{k^{\prime}, l^{\prime}=0}^{N_{x}-1} e^{\frac{2 \pi i}{N_{x}} k k^{\prime}}\left(G_{0 n}\right)_{k^{\prime} l^{\prime}} .
$$



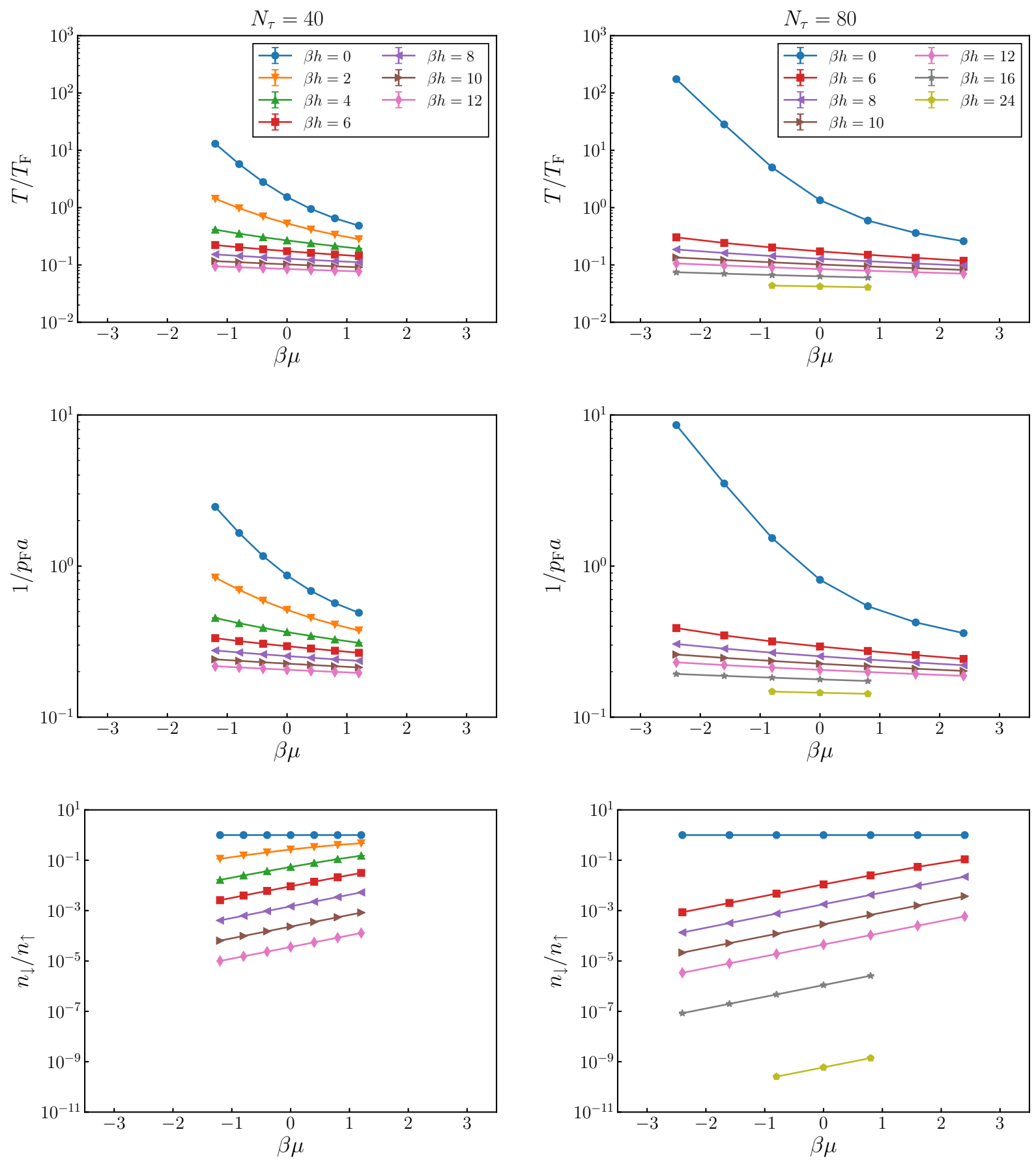

FIG. 3. Dimensionless physical parameters $T / T_{\mathrm{F}}, 1 / p_{\mathrm{F}} a$ and the ratio $n_{\downarrow} / n_{\uparrow}$ of particle numbers for $N_{\tau}=40$ (left) and $N_{\tau}=80$ (right).

\section{NUMERICAL RESULTS}

We performed complex Langevin simulations on $\left(N_{\tau}, N_{x}\right)=(40,60),(80,60)$ lattices. The anisotropy is set to $r=0.1$. There are three dimensionless parameters to characterize the Gaudin-Yang model: $\beta \mu, \beta h$, and $\lambda \equiv \sqrt{\beta} g$. We fixed the dimensionless coupling constant by $\lambda=2$, and swept the average chemical potential and the Zeeman field between $-1.2 \leqslant \beta \mu \leqslant 1.2,0 \leqslant \beta h \leqslant 12$ for $N_{\tau}=40$ and $-2.4 \leqslant \beta \mu \leqslant 2.4,0 \leqslant \beta h \leqslant 24$ for $N_{\tau}=80$, respectively. We set the Langevin step size by $\Delta t=0.01$, and saved configurations of the auxiliary field at the 0.02 interval. For every parameter set, we took 5001 samples. Error bars shown below are $1 \sigma$ statistical errors calculated by the jackknife method, where bin sizes are 0.3-1.2 in units of Langevin time depending on the parameters and observables.

In every Langevin step, the magnitude of the drift term (17) is calculated and stored, and finally the probability distribution $P\left(v^{\eta}\right)$ of the drift term can be drawn. In Fig. 1, a typical result of the probability distribution $P\left(v^{\eta}\right)$ is shown. It is normalized so that the integral of the distribution is 1 . It is empirically known that the drifts distribute over several orders of 
magnitude when the complex Langevin method gives the incorrect results from the previous studies $[26,29,65]$. The drift distribution in Fig. 1 has a limited range $(10 \leqslant v \leqslant 30)$ and does not have a broad tail over several orders of magnitude. Then, we conclude that the simulation by the complex Langevin method gives reliable results. In each simulation, we confirmed that $P\left(v^{\eta}\right)$ shows similar behavior to that in Fig. 1 . This means that our calculation of CLM was reliable.

We also investigated the eigenvalues of the matrix

$$
G_{\sigma, \text { red }}^{-1}=I+e^{N_{\tau} \bar{\mu}_{\sigma}} B^{-1} C_{N_{\tau}-1} \cdots B^{-1} C_{0},
$$

which is the reduced matrix of the inverse Green's function Eq. (28) appearing in the effective action on the lattice Eq. (10) as an effective fermionic matrix. We calculate the eigenvalues $w_{\sigma}$ of the matrix from one configuration in the case of several values of $\beta h=0$ to $\beta h=12$ and other fixed parameters, $N_{\tau}=40$ and $\beta \mu=-1.2$. The imaginary part of $w_{\sigma}$ is negligibly small and hereinafter we discuss the real part of the eigenvalues. The numerical results of eigenvalues $\log \operatorname{Re}\left[w_{\sigma}\right]$ of the matrices $G_{\uparrow, \text { red }}^{-1}$ and $G_{\downarrow \text {,red }}^{-1}$ are shown in Fig. 2. Red circles and blue squares correspond to the eigenvalues of $G_{\uparrow, \text { red }}^{-1}$ and $G_{\downarrow \text {,red }}^{-1}$, respectively. In the case of $\beta h=0$, $w_{\uparrow}$ is exactly the same as $w_{\downarrow}$ because $G_{\uparrow, \text { red }}^{-1}=G_{\downarrow}^{-1}$,red . While the range of the eigenvalues of $G_{\uparrow, \text { red }}^{-1}$ tends to be broad, the range of the eigenvalues of $G_{\downarrow}^{-1}$,red tends to be narrow when $\beta h$ increases.

It is a notable point of this eigenvalue analysis that the eigenvalues of $G_{\sigma \text {,red }}^{-1}$ are always larger than 1 because of $\log (\operatorname{Re}[w])>0$ even in the case of large $\beta h$, corresponding to a large population imbalance. This result indicates that the integrand of the partition function (9) is always positive and no sign problem occurs in the parameter region of our calculations. Note that this is a numerical finding in our setup, and we do not prove that the sign problem never occurs in the Gaudin-Yang model with a population imbalance. We note that the sign problem may occur in other situations within the Hamiltonian (1) or the action (3), for example, considering other values of masses, chemical potentials, coupling constants, lattice parameters, and dimensions.

In Fig. 3, we show the dimensionless quantities $T / T_{\mathrm{F}}$, $1 / p_{\mathrm{F}} a$, and $n_{\downarrow} / n_{\uparrow}$, which are typical indicators of the temperature, the interaction strength, and the population imbalance, respectively. The ratio of particle numbers $n_{\downarrow} / n_{\uparrow}$ becomes significantly small when $\beta h \gg 1\left(\beta \mu_{\uparrow} \gg \beta \mu_{\downarrow}\right)$ as expected. In that case, $T / T_{\mathrm{F}}$ and $1 / p_{\mathrm{F}} a$ are also small since $T_{\mathrm{F}}$ and $p_{\mathrm{F}}$ are proportional to $n_{\uparrow}$.

For each parameter, we computed the ratio of Green's functions $R\left(0, n a_{\tau}\right)$ defined in Eq. (25) at zero momentum. Numerical results on an $N_{\tau}=40$ lattice at $\beta \mu=0$ for a single configuration are shown in Fig. 4. The qualitative behavior of $R\left(0, n a_{\tau}\right)$ at other $N_{\tau}$ and $\beta \mu$ are the same as these results. In the parameter region we swept, $R\left(0, n a_{\tau}\right)$ has a plateau at an intermediate imaginary time, which suggests that the energy spectrum is gapped from any possible excited states. In our analysis, we extract the single-particle ground-state energy $E_{0}(0)$ by

$$
E_{0}(0) \simeq \frac{1}{a_{\tau}} R\left(p, \tau=\left(N_{\tau}-2\right) a_{\tau}\right)
$$

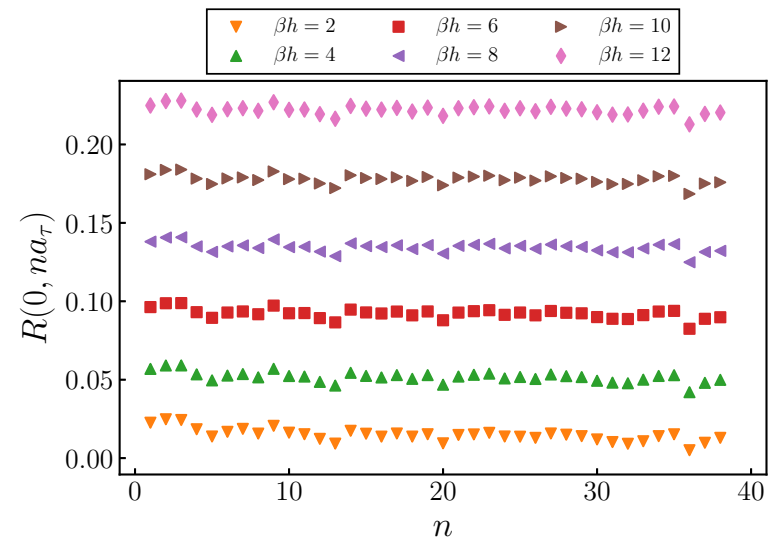

FIG. 4. The $\tau$ dependence of the ratio of Green's functions $R(0, \tau)$ on an $N_{\tau}=40$ lattice at $\beta \mu=0 . n$ denotes the number for the discretized imaginary time $\tau=n a_{\tau}$. Each point in this plot is obtained for a single configuration.

because the long-time limit as Eq. (25) cannot be taken on a lattice.

After calculating the single-particle energy (33), the polaron energy $U$ is obtained by Eq. (26). In Fig. 5, we show the polaron energy on $N_{\tau}=40$ and 80 lattices. As the temporal lattice size $N_{\tau}$ becomes large, the system is close to the continuum limit. The color of each point represents the statistical average of $T / T_{\mathrm{F}}$. The lowest temperature is $T / T_{\mathrm{F}} \simeq 0.08$ for $N_{\tau}=40$ and $T / T_{\mathrm{F}} \simeq 0.07$ for $N_{\tau}=80$, respectively. The ratio of particle numbers $n_{\downarrow} / n_{\uparrow}$ varies from $1.0 \times 10^{-5}$ to $1.5 \times 10^{-1}$ for $N_{\tau}=40$ and from $8.4 \times 10^{-8}$ to $1.0 \times 10^{-1}$ for $N_{\tau}=80$, respectively. The solid line indicates the exact result at zero temperature shown in Eq. (27). For a fixed $N_{\tau}$, the numerical results show similar behavior to Eq. (27) as a function of $1 / p_{\mathrm{F}} a$ despite also depending on $T / T_{\mathrm{F}}$ and $n_{\downarrow} / n_{\uparrow}$. Moreover, the numerical results tend to be close to the exact result at zero temperature when we take the continuum limit. Our result suggests that the polaron energy is insensitive to the temperature and the impurity concentration. Finally, we

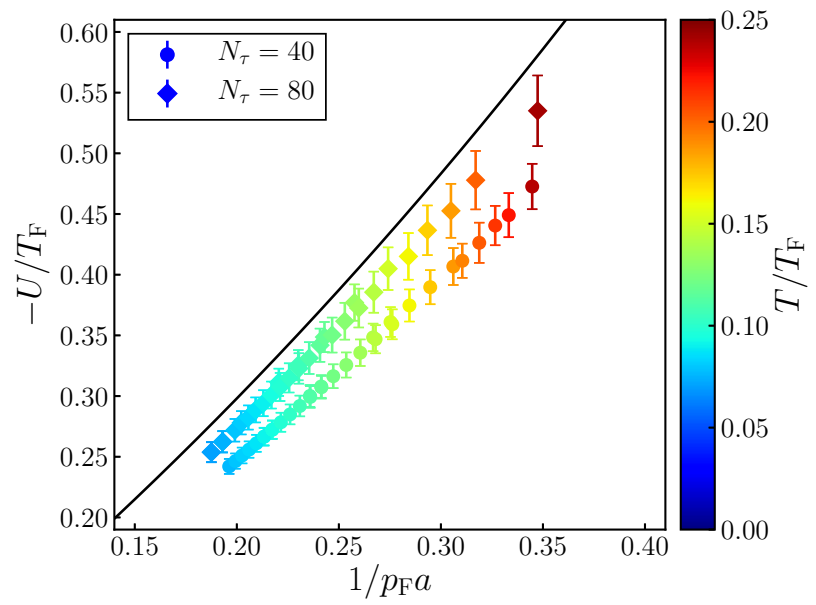

FIG. 5. The polaron energy computed by the CLM. The solid line is the exact result at $T=0$ shown in Eq. (27). 
mention the effective mass of the polaron. While it can be calculated in principle by our setup, the precise calculation was difficult unlike the polaron energy because the several energies at finite momenta of impurity are required in the calculation of the effective mass while only the single energy at zero momentum is required to calculate the polaron energy $U$. If more configurations of the auxiliary fields are generated than our study, the effective mass of the polaron can be precisely calculated.

\section{SUMMARY}

We have studied the excitation properties of Fermi polarons at finite temperature for the attractive Gaudin-Yang model with large population imbalances using the complex Langevin method, a nonperturbative approach free from the sign problem. We have performed numerical simulations for several chemical potentials $\beta \mu$ and Zeeman fields $\beta h$, the dimensionless control parameters of the model, and found that our simulation covers a wide range of temperature $T / T_{\mathrm{F}}$, strength of the coupling $1 / p_{\mathrm{F}} a$, and population imbalance $n_{\downarrow} / n_{\uparrow}$. We have computed the polaron energy as a function of $1 / p_{\mathrm{F}} a$. While our result is still away from the zero temperature and single-polaron limit, the computed polaron energy shows a similar $\left(1 / p_{\mathrm{F}} a\right)$ dependence to the exact result at those limits.

The complex Langevin method works well in the GaudinYang model even in the presence of population imbalance. Practically, within our setup, the probability distribution of the drift term always shows an exponential fall-off, which means that the problem of wrong convergence does not occur. Moreover, the integrand of the path integral is always positive within our simulation from the eigenvalue analysis. However, it is known that the sign problem is severe in the case of higher dimensions [66]. Thus the behavior of the probability distribution of the drift term and the eigenvalues in higher dimensions will be investigated in a future study.

One interesting application of the complex Langevin method is to study the transition from a degenerate Fermipolaron regime to a classical Boltzmann-gas regime of a unitary spin-imbalanced Fermi gas which is found to be a sharp transition by a cold-atom experiment using ${ }^{6} \mathrm{Li} \mathrm{Fermi}$ gases in a three-dimensional box potential [67]. Also, it is interesting to explore an inhomogeneous pairing phase $[36,37]$ and in-medium bound states [68], which cannot be addressed by quantum Monte Carlo simulations due to the sign problem in the mass- and population-imbalanced systems. In order to discuss such phenomena, we need a more elaborate estimation of systematic errors. The work in this direction will be presented elsewhere.

\section{ACKNOWLEDGMENTS}

The authors are grateful to Tetsuo Hatsuda, Kei Iida, and Yuya Tanizaki for fruitful discussions. T.M.D. was supported by a Grant-in-Aid for Early-Career Scientists (No. 20K14480). H.T. was supported by Grants-in-Aid for Scientific Research from JSPS (No. 18H05406). S.T. was supported by the RIKEN Special Postdoctoral Researchers Program. This work was partly supported by RIKEN iTHEMS Program.
[1] W. von der Linden, A quantum Monte Carlo approach to manybody physics, Phys. Rep. 220, 53 (1992).

[2] L. Pollet, Recent developments in quantum Monte Carlo simulations with applications for cold gases, Rep. Prog. Phys. 75, 094501 (2012).

[3] E. Y. Loh, J. E. Gubernatis, R. T. Scalettar, S. R. White, D. J. Scalapino, and R. L. Sugar, Sign problem in the numerical simulation of many-electron systems, Phys. Rev. B 41, 9301 (1990).

[4] M. Troyer and U.-J. Wiese, Computational Complexity and Fundamental Limitations to Fermionic Quantum Monte Carlo Simulations, Phys. Rev. Lett. 94, 170201 (2005).

[5] S. Muroya, A. Nakamura, C. Nonaka, and T. Takaishi, Lattice QCD at finite density: An introductory review, Prog. Theor. Phys. 110, 615 (2003).

[6] P. de Forcrand, Simulating QCD at finite density, PoS 091, 010 (2009) .

[7] E. Vicari and H. Panagopoulos, Theta dependence of SU(N) gauge theories in the presence of a topological term, Phys. Rep. 470, 93 (2009).

[8] W. Krauth, H. Nicolai, and M. Staudacher, Monte Carlo approach to M theory, Phys. Lett. B 431, 31 (1998).

[9] J. Berges, S. Borsányi, D. Sexty, and I.-O. Stamatescu, Lattice simulations of real-time quantum fields, Phys. Rev. D 75, 045007 (2007).
[10] A. Alexandru, G. Basar, P. F. Bedaque, S. Vartak, and N. C. Warrington, Monte Carlo Study of Real Time Dynamics on the Lattice, Phys. Rev. Lett. 117, 081602 (2016).

[11] R. Blankenbecler, D. J. Scalapino, and R. L. Sugar, Monte Carlo Calculations of Coupled Boson-Fermion Systems. I., Phys. Rev. D 24, 2278 (1981).

[12] D. J. Scalapino and R. L. Sugar, Monte Carlo calculations of coupled boson-fermion systems. II, Phys. Rev. B 24, 4295 (1981).

[13] G. Sugiyama and S. Koonin, Auxiliary field Monte-Carlo for quantum many-body ground states, Ann. Phys. 168, 1 (1986).

[14] A. Alexandru, P. F. Bedaque, and N. C. Warrington, Spin polarized nonrelativistic fermions in $1+1$ dimensions, Phys. Rev. D 98, 054514 (2018).

[15] J. E. Hirsch, Two-dimensional Hubbard model: Numerical simulation study, Phys. Rev. B 31, 4403 (1985).

[16] C. Wu and S.-C. Zhang, Sufficient condition for absence of the sign problem in the fermionic quantum Monte Carlo algorithm, Phys. Rev. B 71, 155115 (2005).

[17] Z.-X. Li, Y.-F. Jiang, and H. Yao, Solving the fermion sign problem in quantum Monte Carlo simulations by Majorana representation, Phys. Rev. B 91, 241117(R) (2015).

[18] Z.-X. Li, Y.-F. Jiang, and H. Yao, Majorana-Time-Reversal Symmetries: A Fundamental Principle for Sign-Problem-Free Quantum Monte Carlo Simulations, Phys. Rev. Lett. 117, 267002 (2016). 
[19] Z. C. Wei, C. Wu, Y. Li, S. Zhang, and T. Xiang, Majorana Positivity and the Fermion Sign Problem of Quantum Monte Carlo Simulations, Phys. Rev. Lett. 116, 250601 (2016).

[20] J. R. Klauder, Coherent state Langevin equations for canonical quantum systems with applications to the quantized Hall effect, Phys. Rev. A 29, 2036 (1984).

[21] G. Parisi, On complex probabilities, Phys. Lett. B 131, 393 (1983).

[22] G. Aarts, E. Seiler, and I.-O. Stamatescu, The complex Langevin method: When can it be trusted? Phys. Rev. D 81, 054508 (2010).

[23] G. Aarts, F. A. James, E. Seiler, and I.-O. Stamatescu, Complex Langevin: Etiology and diagnostics of its main problem, Eur. Phys. J. C 71, 1756 (2011).

[24] J. Nishimura and S. Shimasaki, New insights into the problem with a singular drift term in the complex Langevin method, Phys. Rev. D 92, 011501(R) (2015).

[25] K. Nagata, J. Nishimura, and S. Shimasaki, Justification of the complex Langevin method with the gauge cooling procedure, Prog. Theor. Exp. Phys. 2016, 013B01 (2016).

[26] K. Nagata, J. Nishimura, and S. Shimasaki, Argument for justification of the complex Langevin method and the condition for correct convergence, Phys. Rev. D 94, 114515 (2016).

[27] L. L. Salcedo, Does the complex Langevin method give unbiased results? Phys. Rev. D 94, 114505 (2016).

[28] G. Aarts, E. Seiler, D. Sexty, and I.-O. Stamatescu, Complex Langevin dynamics and zeroes of the fermion determinant, J. High Energy Phys. 05 (2017) 044; Erratum to: Complex Langevin dynamics and zeroes of the fermion determinant, 01 (2018) 128 .

[29] K. Nagata, J. Nishimura, and S. Shimasaki, Testing the criterion for correct convergence in the complex Langevin method, J. High Energy Phys. 05 (2018) 004.

[30] M. Scherzer, E. Seiler, D. Sexty, and I.-O. Stamatescu, Complex Langevin and boundary terms, Phys. Rev. D 99, 014512 (2019).

[31] M. Scherzer, E. Seiler, D. Sexty, and I. O. Stamatescu, Controlling complex Langevin simulations of lattice models by boundary term analysis, Phys. Rev. D 101, 014501 (2020).

[32] Z. Cai, X. Dong, and Y. Kuang, On the validity of complex Langevin method for path integral computations, SIAM J. Sci. Comput. 43, A685 (2021).

[33] T. Hayata and A. Yamamoto, Complex Langevin simulation of quantum vortices in a Bose-Einstein condensate, Phys. Rev. A 92, 043628 (2015).

[34] A. C. Loheac, J. Braun, and J. E. Drut, Polarized fermions in one dimension: Density and polarization from complex Langevin calculations, perturbation theory, and the virial expansion, Phys. Rev. D 98, 054507 (2018).

[35] L. Rammelmüller, A. C. Loheac, J. E. Drut, and J. Braun, Finite-Temperature Equation of State of Polarized Fermions at Unitarity, Phys. Rev. Lett. 121, 173001 (2018).

[36] L. Rammelmüller, J. E. Drut, and J. Braun, Pairing patterns in one-dimensional spin- and mass-imbalanced Fermi gases, SciPost Phys. 9, 14 (2020).

[37] L. Rammelmüller, Y. Hou, J. E. Drut, and J. Braun, Pairing and the spin susceptibility of the polarized unitary Fermi gas in the normal phase, Phys. Rev. A 103, 043330 (2021).
[38] A. C. Loheac and J. E. Drut, Third-order perturbative lattice and complex Langevin analyses of the finite-temperature equation of state of nonrelativistic fermions in one dimension, Phys. Rev. D 95, 094502 (2017).

[39] L. Rammelmüller, W. J. Porter, J. E. Drut, and J. Braun, Surmounting the sign problem in nonrelativistic calculations: A case study with mass-imbalanced fermions, Phys. Rev. D 96, 094506 (2017).

[40] C. E. Berger, L. Rammelmüller, A. C. Loheac, F. Ehmann, J. Braun, and J. E. Drut, Complex Langevin and other approaches to the sign problem in quantum many-body physics, Phys. Rep. 892, 1 (2021).

[41] X.-W. Guan, M. T. Batchelor, and C. Lee, Fermi gases in one dimension: From Bethe ansatz to experiments, Rev. Mod. Phys. 85, 1633 (2013).

[42] A. Schirotzek, C.-H. Wu, A. Sommer, and M. W. Zwierlein, Observation of Fermi Polarons in a Tunable Fermi Liquid of Ultracold Atoms, Phys. Rev. Lett. 102, 230402 (2009).

[43] S. Nascimbène, N. Navon, K. J. Jiang, L. Tarruell, M. Teichmann, J. McKeever, F. Chevy, and C. Salomon, Collective Oscillations of An Imbalanced Fermi Gas: Axial Compression Modes and Polaron Effective Mass, Phys. Rev. Lett. 103, 170402 (2009).

[44] M. Koschorreck, D. Pertot, E. Vogt, B. Fröhlich, M. Feld, and M. Köhl, Attractive and repulsive Fermi polarons in two dimensions, Nature (London) 485, 619 (2012).

[45] M. Cetina, M. Jag, R. S. Lous, I. Fritsche, J. T. M. Walraven, R. Grimm, J. Levinsen, M. M. Parish, R. Schmidt, M. Knap, and E. Demler, Ultrafast many-body interferometry of impurities coupled to a Fermi sea, Science 354, 96 (2016).

[46] F. Scazza, G. Valtolina, P. Massignan, A. Recati, A. Amico, A. Burchianti, C. Fort, M. Inguscio, M. Zaccanti, and G. Roati, Repulsive Fermi Polarons in A Resonant Mixture of Ultracold ${ }^{6} \mathrm{Li}$ Atoms, Phys. Rev. Lett. 118, 083602 (2017).

[47] H. S. Adlong, W. E. Liu, F. Scazza, M. Zaccanti, N. D. Oppong, S. Fölling, M. M. Parish, and J. Levinsen, Quasiparticle Lifetime of the Repulsive Fermi Polaron, Phys. Rev. Lett. 125, 133401 (2020).

[48] G. Ness, C. Shkedrov, Y. Florshaim, O. K. Diessel, J. von Milczewski, R. Schmidt, and Y. Sagi, Observation of A Smooth Polaron-Molecule Transition in a Degenerate Fermi Gas, Phys. Rev. X 10, 041019 (2020).

[49] I. Fritsche, C. Baroni, E. Dobler, E. Kirilov, B. Huang, R. Grimm, G. M. Bruun, and P. Massignan, Stability and breakdown of Fermi polarons in a strongly interacting Fermi-Bose mixture, Phys. Rev. A 103, 053314 (2021).

[50] P. Massignan, M. Zaccanti, and G. M. Bruun, Polarons, dressed molecules and itinerant ferromagnetism in ultracold Fermi gases, Rep. Prog. Phys. 77, 034401 (2014).

[51] J. B. McGuire, Interacting fermions in one dimension. II. Attractive potential, J. Math. Phys. 7, 123 (1966).

[52] O. Gamayun, O. Lychkovskiy, and M. B. Zvonarev, Zero temperature momentum distribution of an impurity in a polaron state of one-dimensional Fermi and Tonks-Girardeau gases, SciPost Phys. 8, 53 (2020).

[53] O. Gamayun, A. G. Pronko, and M. B. Zvonarev, Impurity Green's function of a one-dimensional Fermi gas, Nucl. Phys. B 892, 83 (2015). 
[54] O. Gamayun, A. G. Pronko, and M. B. Zvonarev, Time and temperature-dependent correlation function of an impurity in one-dimensional Fermi and Tonks-Girardeau gases as a Fredholm determinant, New J. Phys. 18, 045005 (2016).

[55] W.-B. He, Y.-Y. Chen, S. Zhang, and X.-W. Guan, Universal properties of Fermi gases in one dimension, Phys. Rev. A 94, 031604(R) (2016).

[56] M. Klawunn and A. Recati, Fermi polaron in two dimensions: Importance of the two-body bound state, Phys. Rev. A 84, 033607 (2011).

[57] E. V. H. Doggen and J. J. Kinnunen, Energy and Contact of the One-Dimensional Fermi Polaron at Zero and Finite Temperature, Phys. Rev. Lett. 111, 025302 (2013).

[58] H. Tajima, J. Takahashi, S. I. Mistakidis, E. Nakano, and K. Iida, Polaron problems in ultracold atoms: Role of a Fermi sea across different spatial dimensions and quantum fluctuations of a Bose medium, Atoms 9, 18 (2021).

[59] S. Yadong and Z. Huawen, Polaronic effects in one-dimensional Fermi gas, Eur. Phys. J. D 73, 106 (2019).

[60] S. I. Mistakidis, G. C. Katsimiga, G. M. Koutentakis, and P. Schmelcher, Repulsive Fermi polarons and their induced interactions in binary mixtures of ultracold atoms, New J. Phys. 21, 043032 (2019).
[61] N. Prokof'ev and B. Svistunov, Fermi-polaron problem: Diagrammatic Monte Carlo method for divergent sign-alternating series, Phys. Rev. B 77, 020408(R) (2008).

[62] C. Zhang, N. V. Prokof'ev, and B. V. Svistunov, Peierls/SuSchrieffer-Heeger polarons in two dimensions, Phys. Rev. B 104, 035143 (2021).

[63] C. N. Yang, Some Exact Results for the Many-Body Problem in One Dimension with Repulsive Delta-Function Interaction, Phys. Rev. Lett. 19, 1312 (1967).

[64] M. Gaudin, Un systeme a une dimension de fermions en interaction, Phys. Lett. A 24, 55 (1967).

[65] Y. Ito, H. Matsufuru, Y. Namekawa, J. Nishimura, S. Shimasaki, A. Tsuchiya, and S. Tsutsui, Complex Langevin calculations in QCD at finite density, J. High Energy Phys. 10 (2020) 144.

[66] O. Goulko and M. Wingate, Thermodynamics of balanced and slightly spin-imbalanced Fermi gases at unitarity, Phys. Rev. A 82, 053621 (2010).

[67] Z. Yan, P. B. Patel, B. Mukherjee, R. J. Fletcher, J. Struck, and M. W. Zwierlein, Boiling a Unitary Fermi Liquid, Phys. Rev. Lett. 122, 093401 (2019).

[68] D. Huber, H.-W. Hammer, and A. G. Volosniev, In-medium bound states of two bosonic impurities in a one-dimensional Fermi gas, Phys. Rev. Res. 1, 033177 (2019). 